\section{ALK-positive histiocytosis with KIF5B-ALK fusion in an adult female}

ALK-positive histiocytosis is a relatively recently described entity initially reported only in infants and neonates. ${ }^{1}$ Patients presented with systemic involvement with massive hepatosplenomegaly, bone marrow involvement and cytopenias. Cytologically the cells are reminiscent of juvenile xanthogranuloma (JXG), lacking cellular atypia and anaplasia. More recently, the authors from the original study have reported an expanded confrontation with this rare disease. ${ }^{2}$ Including the cases from their original report, they have identified 10 cases, 9 of which were in infants or children, age 16 or younger. The single case in an adult presented as an isolated breast mass in a 40-year-old female, while the pediatric cases were more likely to have a systemic disease (Table 1). Interestingly, the authors reported the frequent presence of (5 out of 7 cases) a novel KIF $5 B-A L K$ fusion, including the adult patient. In a lesser number of cases the $A L K$ fusion involved other partners, including TPM3 and COLIA2. In this report we describe a case of ALK-positive histiocytosis presenting as acute appendicitis in an adult female; the cells in this lesion also exhibited a $K I F 5 B-A L K$ fusion.

A 50-year-old Caucasian female presented with right lower quadrant pain (7 out of 10 on the standard pain scale) and pressure with urination. The urine analysis was negative. A contrast enhanced (CT) scan of the abdomen and pelvic showed findings most consistent with acute appendicitis. A laparoscopic appendectomy was performed. The appendix contained a $1.5 \times 1.5 \mathrm{~cm}$ circumferential nodule in the mid portion of the appendix, partially obliterating the intestinal lumen (Figure 1A). Histologic sections showed an atypical infiltrate consisting of round to oval, lobulated, and focally spindle shaped cells with vesicular chromatin, basophilic nucleoli and abundant pale eosinophilic cytoplasm (Figure $1 \mathrm{~B}, \mathrm{C}$ ). The infiltrate extended from the mucosa through the muscularis, with focal infiltration of the surrounding adipose tissue. Due to luminal obstruction, portions of the remaining appendix showed acute appendicitis and serositis. A panel of immunostains [CD30, CD21, CD23, ALK1, SMA, CD117, DOG1, CD68, CD1a, S-100, desmin, CD163, EMA, AE1/AE3, CD4, CAM5.2, CD3, CD2, TIA1, granzyme B, myeloperoxidase] and EpsteinBarr virus encoded-RNA in situ hybridization (EBER ISH)] were performed. The atypical polygonal and spindleshaped cells were positive for CD4, CD163, (Figure 1 E,
F) and CD68, consistent with histiocytes. An ALK1 immunostain showed cytoplasmic positivity in the atypical cells (Figure 1D). The atypical cells were negative for CD1a, S-100, CD117, DOG1, SMA, desmin, EMA, CD30, CD3, CD2, MPO, CD21, CD23, CAM5.2, AE1/AE3. The EBER ISH was negative. Other stains were non-contributory.

Positive staining for ALK prompted further genetic studies. Fluorescence in situ hybridization (FISH) for $A L K$ rearrangement (Vysis LSI Dual Color ALK break apart probe, Abbott Molecular, Inc.) performed on formalinfixed paraffin-embedded (FFPE) tissue sections was positive for $A L K$ rearrangement (Figure $1 \mathrm{G}$ ) with split signals in $80 \%$ of the nuclei. In order to identify the fusion partner, a next-generation sequencing based assay (Oncomine $^{\mathrm{TM}}$ Comprehensive Assay v3; Thermo Fisher Scientific) was performed as described previously. ${ }^{3}$ An $A L K-K I F 5 B$ fusion (COSMIC ID: COSF1058) was detected joining exon 20 of ALK and exon 24 of KIF5B. The fusion sequencing reads were reviewed in Integrative Genomics Viewer (IGV) (Figure 1H).

The overall morphological, immunophenotypical and molecular findings in this case confirmed a diagnosis of ALK-positive histiocytosis, as described by Chang et al. in their recent series. ${ }^{2}$ While the differential diagnosis included an inflammatory myofibroblastic tumor (IMT), the atypical cells were negative for SMA, and the immunophenotype indicated a histiocytic derivation. The bland cytological features did not suggest a histiocytic sarcoma. Importantly, this case confirms that the diagnosis of ALK-positive histiocytosis should be considered for all histiocytic lesions, whether in children or adults.

Most cases of ALK-positive histiocytosis with a $K I F 5 B-A L K$ fusion appear to have an indolent clinical course, with limited data on the role of chemotherapy. However, Chang et al. reported a clinical response to crizotinib, an ALK inhibitor, in an unresectable cavernous sinus lesion with complete resolution at 3 months. Additionally, a 40-year-old male with widespread "nonLangerhans cell histiocytosis" showed a marked response to a crizotinib-based treatment regimen. ${ }^{4}$ Therefore, the identification of the KIF $5 B-A L K$ fusion may have significant therapeutic implications.

$A L K$ gene fusions may involve multiple different fusion partners and have been reported in various epithelial and mesenchymal malignancies. ${ }^{4}$ Among all neoplasms, they are relatively common in non-small cell carcinoma of the lung. However, the novel KIF5B-ALK gene fusion identified by Chang et al. ${ }^{2}$ and identified in other rare histiocytic lesions ${ }^{4,5}$ appears to be a highly recurrent feature of

Table 1. Clinical features of ALK-positive histiocytosis with KIF5B-ALK fusion.

\begin{tabular}{lccccc}
\hline Reference & Age & Sex & Ethnicity & Sites of involvement & Therapy and follow Up \\
2 & 2 months & F & Asian & Systemic & ChemoRx; NED at 2 years \\
2 & 3 months & M & Caucasian & Systemic & ChemoRx; Limited skin nodules at 4 years \\
\hline 2 & 2 years, 3 months & M & Caucasian & Localized skin & Excision; NED at 2.5 years \\
2 & 15 years & M & Caucasian & Cavernous sinus & ALK-inhibitor; NED \\
\hline 2 & 40 years & F & Asian & Localized breast & Excision; NED at 3.5 years \\
4 & 40 years & M & NS & Multiple extranodal & ChemoRx; ALK-inhibitor; Prolonged PR \\
\hline 5 & 25 years & NS & NS & Localized skin & NS \\
5 & 50 years & NS & NS & Localized liver & NS \\
\hline current & 50 years & F & Caucasian & Appendix & Excision; NED (limited follow up) \\
\hline
\end{tabular}

NS: not stated; ChemoRX: chemotherapy; NED: no evidence of disease; PR: partial remission. 

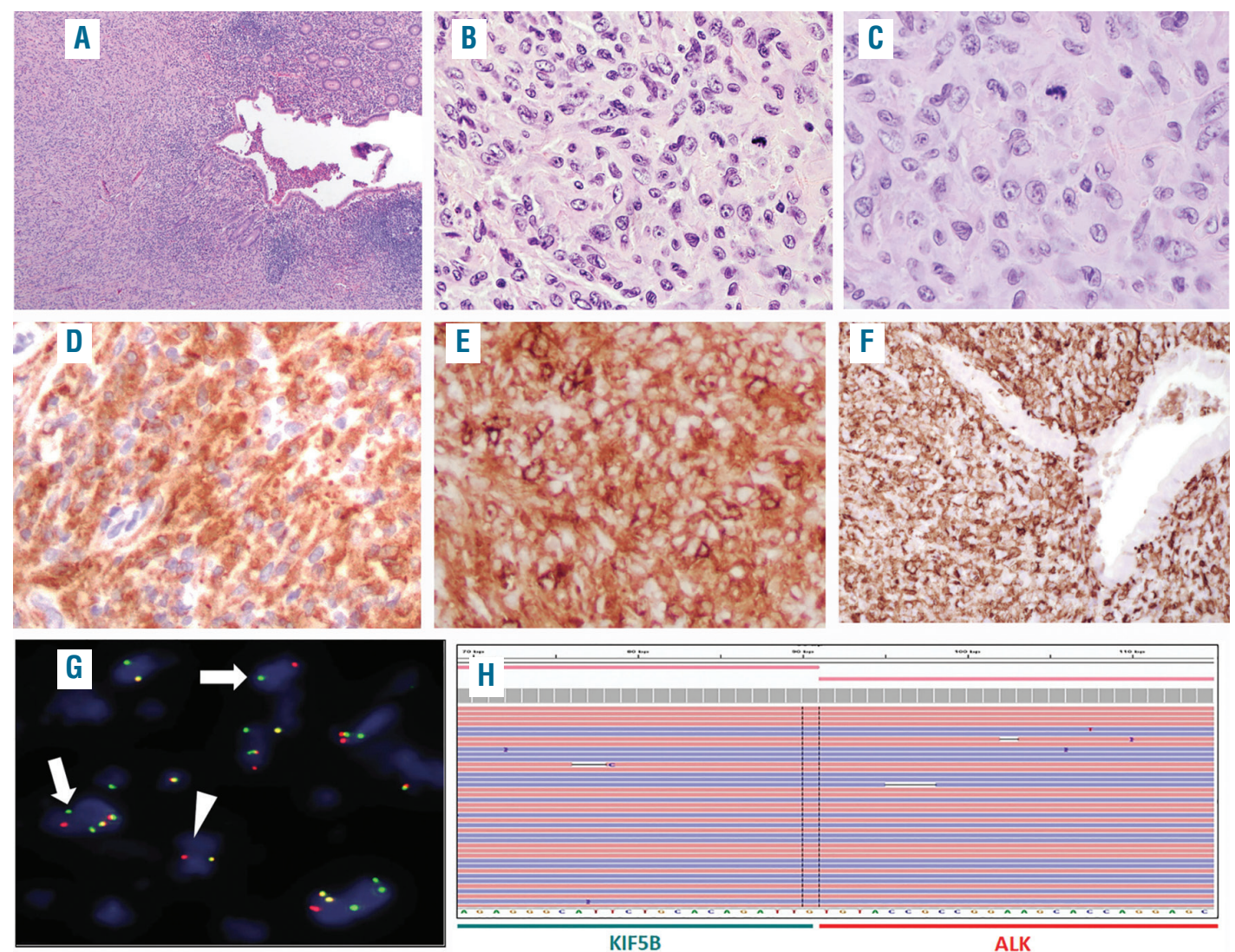

Figure 1. Morphologic, immunophenotypic and molecular features of ALK-positive histiocytosis. (A) Appendix diffusely infiltrated by an atypical infiltrate partially obliterating the intestinal lumen (100X); (B) Atypical cells show varied nuclear contours with absence of eosinophils and plasma cells (400x). (C) Occasional mitotic figures were seen. (600x). (D) Atypical cells were positive with the ALK1 IHC stain (200 X); (E) CD4 IHC stain (200 X); and F) CD163, which shows atypical cells extending to entrapped glandular epithelium at the luminal surface; IHC stain (200X); (G) Fluorescence in situ hybridization showing ALK rearrangement. Nuclei (DAPI) with split apart signal (individual green and red signal; arrow) and or single red signal (deleted green signal; arrowhead) in addition to fused and/or split red/green signals are ALK rearrangement positive. (H) Integrative Genomic Viewer (IGV) window snapshot exhibiting the ALK (exon 20)-KIF5B (exon 24) RNA fusion.

ALK-positive histiocytosis. Notably, this fusion has been seen only rarely in lung cancer. ${ }^{6}$

A significant issue is the exact designation of the histiocytic neoplasms associated with the KIF5B-ALK fusion. In a study of diverse forms of histiocytosis, Diamond et al. identified the KIF5B-ALK fusion in 2 out of 24 patients, both of whom were considered to have $B R A F^{\mathrm{V} 600 \mathrm{E}}$-negative Erdheim-Chester disease (ECD). ${ }^{5}$ However, neither patient had the characteristic bone lesions, seen in approximately $95 \%$ of patients with ECD.' Both were adults (25 and 50 years of age) with localized disease involving the skin and liver respectively. Ross et al. reported an additional fusion positive case in a 40-year- old male with widespread disease involving the liver, omentum, skin, and bone. ${ }^{4}$ Overall, cases of ALKpositive histiocytosis with the KIF $5 B-A L K$ fusion fall into two clinical scenarios: infants with a disseminated but generally self-limited disease, and older children and adults who tend to have single or multiple lesions involving a variety of extranodal sites.

The remaining issue is the preferred nomenclature for this neoplasm. Notably, it was not specifically designated in the recently published World Health Organization classification. ${ }^{8}$ Emile et al. in their classification included similar cases within the category of ECD, with the designation "extracutaneous or disseminated JXG with MAPK-activating mutation or ALK translocations".? However, as noted by us and other authors, these cases differ significantly from both ECD and JXG. We believe the emerging data provide sufficient evidence for the designation of ALK-positive histiocytosis as a separate disease that is highly associated with the KIF5B-ALK fusion. The more widespread testing of histiocytic lesions for ALK in both children and adults may provide further insight into this unusual neoplasm.

Gaurav K. Gupta, Liqiang Xi, ${ }^{1}$ Svetlana D. Pack, Jennifer B. Jones, ${ }^{2}$ Stefania Pittaluga, ${ }^{1}$ Mark Raffeld' and

Elaine S. Jaffe ${ }^{1}$

'Laboratory of Pathology, Center for Cancer Research, National Cancer Institute, National Institutes of Health, Bethesda, MD and 2Prisma Health-Midlands, Sumter, SC, USA

Correspondence: ELAINE S. JAFFE.

ejaffe@mail.nih.gov

doi:10.3324/haematol.2019.230094

Funding: this work was supported by the intramural research program of the Center for Cancer Research, National Cancer Institute, National Institutes of Health. 


\section{CASE REPORTS}

Information on authorship, contributions, and financial \& other disclosures was provided by the authors and is available with the online version of this anticle at www. haematologica.org.

\section{References}

1. Chan JK, Lamant L, Algar E, et al. ALK+ histiocytosis: a novel type of systemic histiocytic proliferative disorder of early infancy. Blood. 2008:112(7):2965-2968.

2. Chang KTE, Tay AZE, Kuick CH, et al. ALK-positive histiocytosis: an expanded clinicopathologic spectrum and frequent presence of KIF5B-ALK fusion. Mod Pathol. 2019;32(5):598-608.

3. Luthra R, Patel KP, Routbort MJ, et al. A Targeted high-throughput next-generation sequencing panel for clinical screening of mutations, gene amplifications, and fusions in solid tumors. J Mol Diagn. 2017; 19(2):255-264.
4. Ross JS, Ali SM, Fasan O, et al. ALK Fusions in a wide variety of tumor types respond to anti-ALK targeted therapy. Oncologist. 2017; 22(12):1444-1450

5. Diamond EL, Durham BH, Haroche J, et al. Diverse and targetable kinase alterations drive histiocytic neoplasms. Cancer Discov. 2016; 6(2):154-165.

6. Takeuchi K, Choi YL, Togashi Y, et al. KIF5B-ALK, a novel fusion oncokinase identified by an immunohistochemistry-based diagnostic system for ALK-positive lung cancer. Clin Cancer Res. 2009;15(9):3143-3149.

7. Emile JF, Abla O, Fraitag S, et al. Revised classification of histiocytoses and neoplasms of the macrophage-dendritic cell lineages. Blood. 2016;127(22):2672-2681.

8. Swerdlow SH, Campo E, Harris NL, et al., eds. WHO classification of tumours of haematopoietic and lymphoid tissues revised $4^{\text {th }}$ Edition ed. Lyon, France: International Agency for Research on Cancer (IARC) 2017. 